\section{Tuberculosis in non-human primates of Assam: use of PrimaTB STAT-PAK Assay for detection of tuberculosis}

\section{Bichitra Gopal Nath ${ }^{1}$, Apurba Chakraborty ${ }^{2}$ \& Taibur Rahman ${ }^{3}$}

${ }^{1}$ Research Associate,ICAR-RC for NEH Region, Sikkim Centre, Tadong, Sikkim 737102, India

${ }^{2}$ Director of Research (Veterinary), ${ }^{3}$ Professor, Department of Pathology, College of Veterinary Science, Assam Agricultural University, Khanapara, Guwahati, Assam 781022, India Email: ${ }^{1}$ drbichitra.nath@gmail.com (corresponding author) ${ }^{2}$ drapurba2@gmail.com, ${ }^{3}$ drtaibur.rahman@gmail.com

Tuberculosis (TB) is an important bacterial disease of non-human primates and remains a serious threat to the health of captive non-human primates as well as their animal keepers. The danger of this disease lies in its frequency of occurrence, its ability to spread rapidly, its high mortality rates and zoonotic potential (Garcia et al. 2004a; Vervenne et al. 2004). Tuberculosis in nonhuman primates is caused by the same organisms that are responsible for tuberculosis in humans (Mycobacterium tuberculosis) and cattle (Mycobacterium bovis) (Capuano et al. 2003; Flynn et al. 2003).

The study also screened three free ranging nonhuman primates of Kaziranga National Park for TB

Date of publication (online): 26 April 2012

Date of publication (print): 26 April 2012

ISSN 0974-7907 (online) | 0974-7893 (print)

Editor: Ulrike Streicher

\section{Manuscript details:}

Ms \# 02860

Received 02 July 2011

Final received 05 January 2012

Finally accepted 13 February 2012

Citation: Nath, B.G., A. Chakraborty \& T. Rahman (2012). Tuberculosis in non-human primates of Assam: use of PrimaTB STAT-PAK Assay fo detection of tuberculosis. Journal of Threatened Taxa 4(4): 2541-2544.

Copyright: @ Bichitra Gopal Nath, Apurba Chakraborty \& Taibur Rahman 2012. Creative Commons Attribution 3.0 Unported License. JoTT allows unrestricted use of this article in any medium for non-profit purposes, reproduction and distribution by providing adequate credit to the authors and the source of publication.

Acknowledgement: The authors are grateful to Dr. Prasanta Boro (Veterinary Officer), WTI for providing the serum samples and the authority of Assam State Zoo and Department of Forest and Environment, Govt. of Assam for the materials and the Head, Department of Pathology, College of Veterinary Science, Assam Agricultural University, Khanapara for providing the facilities.

OPEN ACGESS | FREE DOWNLOAD by using Prima TB STAT-PAK Assay kit. PrimaTB STAT-PAK is based on lateral flow technology,

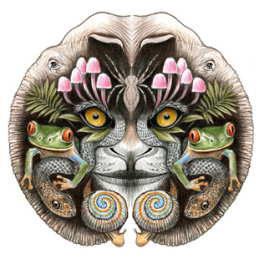
a simple screening assay with highly sensitive and highly specific results. It is an easy-to-perform disposable kit which can use serum, plasma, or fresh whole-blood samples to provide yes or no results within 15 to 20 minutes. The complete test is easy and convenient to administer, and is less traumatic to the animals. When used alone or in combination with tuberculin skin testing, Prima TB STAT-PAK helps reduce TB transmission.

Materials and methods: A total of 27 carcasses of non-human primates of Assam State Zoo and Department of Forest and Environment, Government of Assam were studied from December 2007 to November 2009. The authority of zoo and forest department had submitted the carcasses to the Pathology Department, College of Veterinary Science, Assam Agricultural University, Khanapara for necropsy to determine the cause of death. Postmortem examinations were conducted, the gross lesions were recorded and tissue samples were preserved in 10\% formaldehyde and then processed and stained by routine pathohistological methods with haematoxyline and eosin. From the affected organs, duplicate sections were also stained with special techniques such as Brown and Brenn, Ziehl-Neelsen and modified Periodic Acid Schiff. Smears were prepared from the caseous material inside the tuberculous structures and were also stained by Ziehl-Neelsen's technique.

Three serum samples were collected from free ranging non-human primates of Kaziranga National Park. These animals were found injured and had been caught and tranquilized for treatment by the assistants of Wildlife Trust of India (WTI). Blood/serum samples were collected from these animals for different diagnostic tests. Those three serum samples were tested for TB by using Prima TB STAT-PAK Assay kit. This ready-to-use assay includes a disposable plastic device. A unique cocktail of Mycobacterium tuberculosis and Mycobacterium bovis antigens combine with blue latex beads that adhere to the sample if the antibody is present. A blue band will be visible in the Test (T) window signaling a reactive result. A blue band in the Control (C) window indicates a valid test. No band will appear in the Test $(\mathrm{T})$ window if the results are 


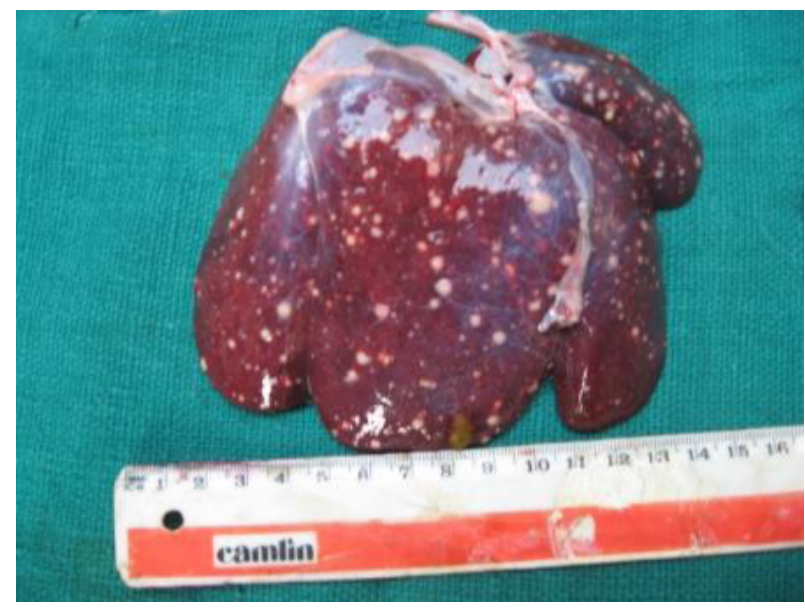

Image 1. Liver showing tuberculus lesion

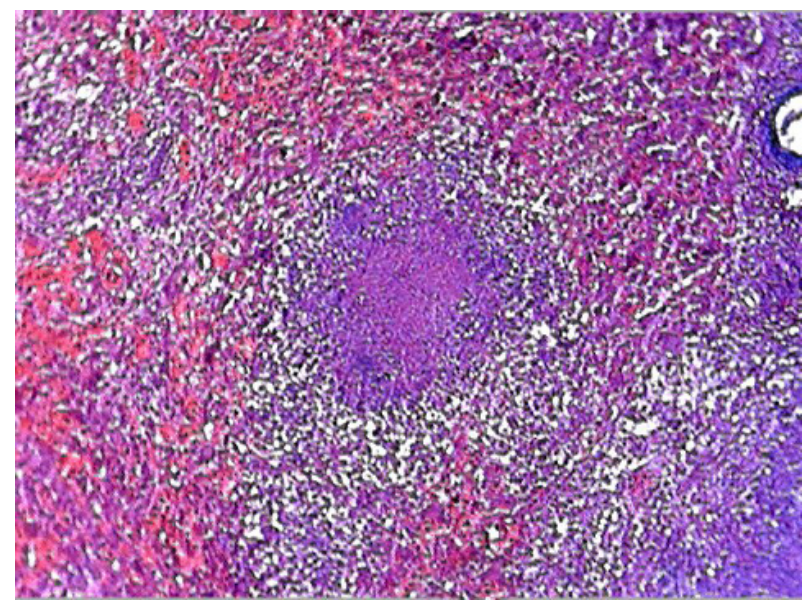

Image 3. Photomicrograph of liver showing tuberculus granuloma H\&E X 100.

non-reactive.

Result: Off the 27 non-human primate carcasses examined, six were found to be positive for tuberculosis. Macroscopic tuberculous lesions were seen in the liver (Image 1), lungs and spleen (Image 2). The other organs like lymph nodes and kidneys also showed lesions but to a lesser degree. The lesion consisted of numerous yellowish-white nodules of varying size ranging from 1 to $10 \mathrm{~mm}$ in diameter. Incision of the nodular growth revealed thick caseous material, which contained numerous acid fast organisms. Microscopically, the liver showed multiple tuberculous granuloma (Image 3) distributed throughout the hepatic parenchyma. The granulomes were characterized by cellular debris surrounded by macrophages, epitheloid cells and giant cells (Image 4) with mild proliferation of connective tissue. The interlobular septa were thickened with

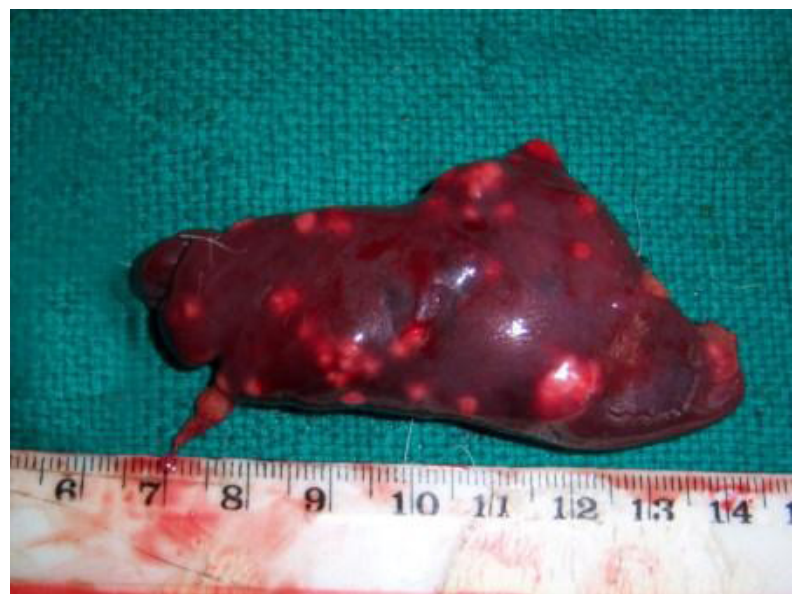

Image 2. Spleen showing tuberculus lesion

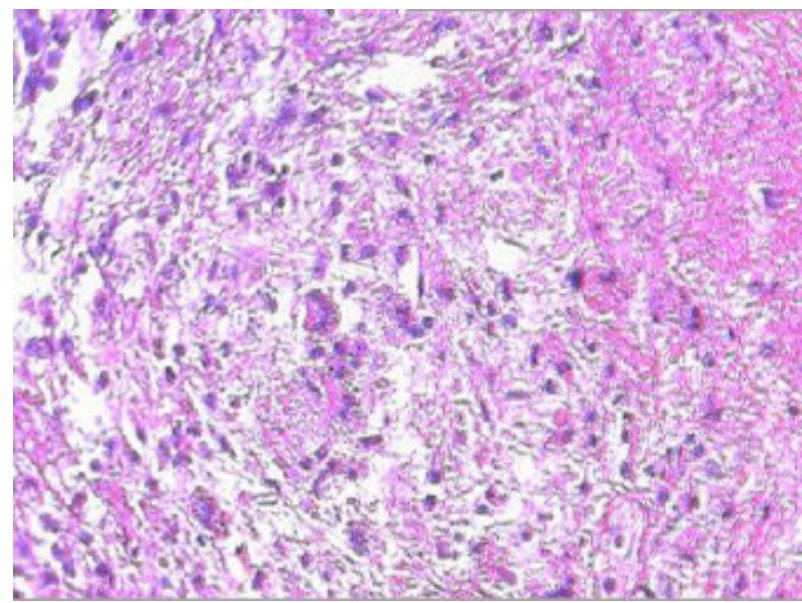

Image 4. Photomicrograph of liver showing giant cell H\&E $\mathrm{X} 400$

fibrinous exudates along with neutrophils. In the lung parenchyma tuberculous granuloma showed a central area of necrosis with cellular debris surrounded by a large number of epitheloid cells, lymphocytes and Langhan's giant cells encapsulated by connective tissue. The spleen showed a central large area of necrosis with cellular debris surrounded by a large number of epitheloid cells, Langhan's giant cells and lymphocytes surrounded by a capsule of connective tissue. Small acid fast organisms were found in the cytoplasm or in the epitheloid cells of the affected organs.

PrimaTB STAT-PAK Assay for detection of Tuberculosis: All three samples showed negative result (Image 5) indicating the serum did not have antibodies to Mycobacterium tuberculosis or Mycobacterium bovis. 


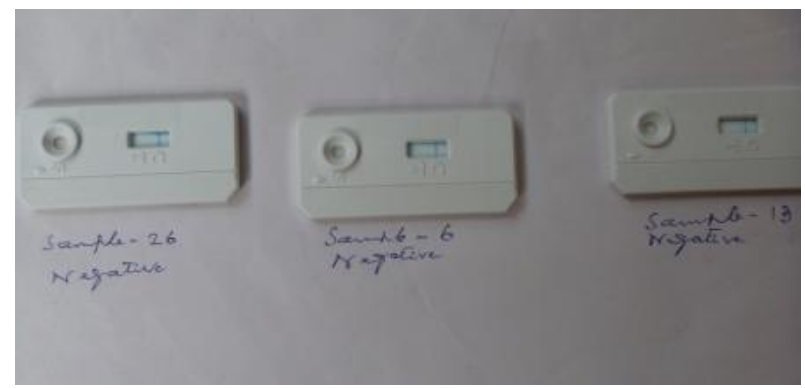

Image 5. Negative result in the PrimaTB STAT-PAK Assay for detection of antibodies to Mycobacterium spp. (note: absence of any band in the 'T' region)

Discussion: Tuberculosis is a common and a major cause of death in non-human primates (Goswami 1994; Goswami \& Chakraborty 1996; Martino et al. 2007). The macroscopic and histopathological alterations found in this study were similar to those described by the other researchers. The postmortal study confirmed the earlier report that tuberculosis is widely spread in captive animals and therefore a matter of concern. In the present study, as only carcasses were supplied to the department of pathology, so we did not get the chance to detect TB from blood/serum of animals prior to death which would help us to confirm the diagnosis from both blood/serum test and post mortem lesion.

Although the intradermal tuberculin skin test (TST) has been a valuable tool in efforts to control tuberculosis in nonhuman primates, but it gives only intermittently positive results in serial testing of infected animals (Garcia et al. 2004b) and for TSTpositive infected animals (even those with radiographic evidence of lung disease) to eventually give negative TST results on serial testing due to the development of latent infections, anergy associated with progressive disease, or other mechanisms that are poorly understood (Motzel et al. 2003). Recent advances in the sequencing of the M. tuberculosis genome and the development of multiplex microbead assays (MMIA) have provided the opportunity to simultaneously determine antibody profiles against multiple $M$. tuberculosis antigens. Investigators have reported antibody responses to recombinant antigens unique to M. tuberculosis in infected macaques (Brusasca et al. 2003), as well as the value of antibody profiles in humans against multiple $M$. tuberculosis antigens in TB serodiagnosis and disease states (Davidow et al. 2005). Another assay format that allows for the simultaneous evaluation of the pattern of reactivity to multiple TB-specific antigens is the multiantigen print immunoassay (MAPIA) (Lyashchenko et al. 2000). In this assay, multiple antigens are immobilized in solid phase on nitrocellulose membranes as narrow bands using a semi-automated airbrush printing device. Presence of a visible band is interpreted as a positive result (Lyashchenko et al. 2000).

The Prima TB STAT PAK is an improvement over the currently common intradermal tuberculin test because it produces results within 15-20 minutes instead of the currently required 72 hours of repeated observations and requires a small volume $(\sim 30 \mu \mathrm{l})$ of serum, plasma, or whole-blood. The rapid test can be performed cage-side and does not require laboratory equipment or specific technical training. This assay uses a "cocktail" of three immunodominant TBspecific antigens (ESAT-6, CFP-10, and MPB83), a combination that was sufficient to correctly identify 25 of 27 (93\%) macaques (20 rhesus and 7 cynomolgus) experimentally infected with $M$. tuberculosis or $M$. bovis and testing of 195 uninfected macaques produced three $(1.5 \%)$ false positive results (Greenwald et al. 2007).

To control the spread of the disease, captive nonhuman primates should be screened regularly. By using Prima TB STAT PAK Assay kit, the animals which are found positive should be quarantined and treated resulting in reduction in the spread of the disease to other healthy animals. Animals infected with TB may not show symptoms of disease for weeks or months (Gibson 1998), but during that time they can spread the infection to other animals. Detection of latent TB infections is therefore a high priority in the control and prevention of the disease in nonhuman primates. The study suggests that colony managers and zoo authorities should screen the animals regularly to control the spread of this infectious disease.

\section{REFERENCES}

Brusasca, P.N., R.L. Peters, S.L. Motzel, H.J. Klein \& M.L. Gennaro (2003). Antigen recognition by serum antibodies in non-human primates experimentally infected with Mycobacterium tuberculosis. Comparative Medicine 53: $165-172$.

Capuano,S.V.,III,D.A. Croix,S.Pawar,A.Zinovik, A. Myers, P.L.Lin,S.Bissel,C.Fuhrman,E.Klein \&J.L.Flynn(2003). 
Experimental Mycobacterium tuberculosis infection of cynomolgus macaques closely resembles the various manifestations of human M.tuberculosis infection. Infection and Immunity 71: 5831-5844.

Davidow, A., G.V. Kanaujia, L. Shi, J. Kaviar, X.S. Guo, G. Kaplan, D. Menzies \& M.L. Gennaro (2005). Antibody profiles characteristic of Mycobacterium tuberculosis infection state. Infection and Immunity 73: 6846-6851.

Flynn, J.L., S.V. Capuano, D. Croix, S. Pawar, A. Myers, A. Zinovik \& E. Klein (2003). Non-human primates: a model for tuberculosis research. Tuberculosis 83: 116-118.

Garcia, M.A., D.M. Bouley, M.J. Larson, B. Lifland, R. Moorhead, M.D. Simkins, D.C. Borie, R. Tolwani \& G. Otto (2004a). Outbreak of Mycobacterium bovis in a conditioned colony of rhesus (Macaca mulatta) and cynomolgus(Macaca fascicularis) macaques. Comparative Medicine 54: 578-584.

Garcia, M.A., J. Yee, D.M. Bouley, R. Moorhead \& N.W. Lerche (2004b). Diagnosis of tuberculosis in macaques, using whole-blood in vitro interferon-gamma (PRIMAGAM) testing. Comp Med. 54: 86-92.

Gibson, S. (1998). Bacterial and Mycotic Diseases: Non-human Primates in Biomedical Research. San Diego: Academic Press, 59-110pp.

Goswami, P.K. (1994). Studies on prevalence of pathological conditions of captive non human primate. MVSc Thesis. Assam Agricultural University.

Goswami, P.K. \& A. Chakraborty (1996). Studies on prevalence of pathological conditions of captive non human primates. Zoos' Print 11(10): 10.

Greenwald, R., K. Lyashchenko, J. Esfandiari, S. Gibson, P. Didier, C. McCombs \& L. Stutzman (2007). PrimaTB STAT-PAK ${ }^{\mathrm{TM}}$ assay, a novel rapid test for tuberculosis in nonhuman primates. American Journal of Primatology 69(1): 120.

Lyashchenko, K.P., M. Singh, R. Colangeli \& M.L. Gennaro (2000). A multi-antigen print immunoassay for the development of serological diagnosis of infectious diseases. Journa of Immunological Methods 242: 91-100.

Martino, M., G.B. Hubbard \& N. Schlabritz-Loutsevitch (2007). Tuberculosis (Mycobacterium tuberculosis) in a pregnant Baboon (Papio cynocephalus). Journal of Medical Primatology 36(2): 108-112.

Motzel, S.L., R.D. Schachner, R.W. Kornegay, M.A. Fletcher, B. Kanaya, J.A. Gomez, DT-W. Ngai, W.J. Pouch, M.V. Washington, L.A. Handt, J.L.Wagner \& H. Klein (2003). Diagnosis of tuberculosis in nonhuman primates, pp. 156-159. In: International Perspectives: The Future of Nonhuman Primate Resources. Washington: National Academies Press.

Vervenne, R.A., S.L. Jones, D. van Soolingen, T. van der Laan, P. Andersen, P.J. Heidt, A.W. Thomas \& J.A. Langermans (2004). TB diagnosis in non-human primates: comparison of two interferon-gamma assays and the skin test for identification of Mycobacterium tuberculosis infection. Veterinary Immunology and Immunopathology 100: 6171 . 